Research Article

\title{
Fault Diagnosis and Classification in Urban Rail Vehicle Auxiliary Inverter Based on Wavelet Packet and EIman Neural Network
}

\author{
Dechen Yao ${ }^{1, *}$, Limin $\mathrm{Jia}^{1}$, Changxu Ji ${ }^{1}$, Yong Qin ${ }^{1}$, Guangwu Liu ${ }^{2}$ and Shiyou Zhu ${ }^{2}$ \\ ${ }^{I}$ State Key Laboratory of Rail Traffic Control and Safety, Beijing Jiaotong University Beijing, China \\ ${ }^{2}$ Guangzhou Metro.Guangzhou, 510030, China
}

Received 25 June 2012; Accepted 15 January 2013

\begin{abstract}
In this paper we present a novel method in fault recognition and classification in urban rail vehicle auxiliary inverter based on wavelet packet and Elman neural network. First, the original fault voltage signals are decomposed by wavelet packet. Next, an automatic feature extraction algorithm is constructed. Finally, those wavelet packet energy eigenvectors are used as Elman neural network input parameters to realize intelligent fault diagnosis. The result shows that the Elman neural network is better than BP neural network, it is effective to distinguish the state of the urban rail vehicle auxiliary inverter.
\end{abstract}

Keywords: Fault Diagnosis, Urban Rail Vehicle Auxiliary Inverter, Wavelet Packet, Elman Neural Network

\section{Introduction}

Auxiliary inverters are widely used in urban rail vehicle, according to statistics, most of the electrical fault is caused by auxiliary inverter. Once the auxiliary inverter's fault, it causes both personal damage and heavy economic loss. Therefore, monitoring and fault diagnosis of the auxiliary inverter is essential.

Numerous condition monitoring and diagnostics methodologies are utilizing to identify the faults of auxiliary inverter. For example, FFT, STFT, wavelet transform, neutral networks [1-3] and soon. The FFT has drawback, when signal is nonstationary or noisy, even in FFT, time information is lost. Many researchers have used short-time Fourier transform (STFT) to overcome the time information problem but low resolution problem exists in STFT. The wavelets transform is currently used to overcome both the time information and low resolution problems. A major advantage of the wavelets transform is that this method can exhibit the local features of the signals and give account of how energy is distributed over frequencies changes from one instant to the next [4]. Wavelet packet transform (WPT) is an important extension of wavelet transform. Wavelet packet (WP) functions inherit the property of time-frequency localization from their corresponding wavelet functions, but offer more flexibility than wavelet in representing different type of signals. Comparing to wavelet analysis, WPT has found a few applications in chemistry, and is still rare used in the pre-processing of ANN input data [5]. In the recent years, the artificial neural network has been widely applied on fault diagnosis. ANNs are nonlinear methods which

*E-mail address: shmily137@sina.com ISSN: 1791-2377 @ 2013 Kavala Institute of Technology. All rights reserved. mimic nerve system. They have functions of self-organizing, data-driven, self-study, self-adaptive and associated memory. ANNs can learn from patterns and capture hidden functional relationships in a given data even if the functional relationships are not known or difficult to identify. Using the training methods, an ANN can be trained to identify the underlying correlation between the inputs and outputs. Later, the unseen inputs can be fed to the trained ANN to generate appropriate outputs [6]. In this study, recurrent neural network approach, which is named as Elman's net, is used for monitoring of the urban rail vehicle auxiliary inverter. This paper aims to propose an effective fault diagnosis and classification method for urban rail vehicle auxiliary inverter based on wavelet packet and Elman neural network. The feasibility of this method is demonstrated through the data of voltage signal. These results reported in this paper clearly demonstrate the accuracy of implementing this approach for urban rail vehicle auxiliary inverter.

The paper is organized as follows. Section 2 briefly discusses the wavelet and wavelet packet. section 3 describes the wavelet packet energy vector algorithm. Section 4 illustrates Elman neural networks. Section 5 demonstrates the effectiveness of the proposed wavelet packet and Elman neural network algorithm for faults diagnosis and classification in urban rail vehicle auxiliary inverter. Finally, conclusions are given in Section 6.

\section{Wavelet and wavelet packet transform}

If $\psi(t) \in L^{2}(R)$ and its Fourier transform, $\hat{\psi}(f)$, satisfy the admissibility condition [7] 


$$
C_{\psi}=\int_{-\infty}^{\infty} \frac{|\hat{\psi}(f)|^{2}}{|f|}<\infty
$$

A wavelet $\psi(t)$ is a function of zero average

$$
\int_{-\infty}^{\infty} \psi(t)=0
$$

$\psi(t)$ is a wavelet function and $L^{2}(R)$ is the space of square integrable complex functions. The corresponding family of wavelets consists of a series of son wavelets, which are generated by dilation and translation from the mother wavelet $\psi(t)$, is shown as follows:

$\psi_{a, b}=\frac{1}{\sqrt{a}} \psi\left(\frac{t-b}{a}\right) \quad a, b \in R, a \neq 0$

where $\mathrm{a}$ and $\mathrm{b}$ are the dilation and translation parameters, respectively.

Wavelet packet analysis method is the promotion of multi-resolution wavelet analysis, simply, it is a function family. First, the two-scale relations of the orthogonal scaling function $\varphi(t)$ and wavelet function $\psi(t)$ are as follows [8]:

$$
\begin{aligned}
& \varphi(t)=\sqrt{2} \sum_{k} h_{0 k} \varphi(2 t-k) \\
& \psi(t)=\sqrt{2} \sum_{k} h_{1 k} \psi(2 t-k)
\end{aligned}
$$

( $h_{0 k}$ and $h_{1 k}$, the filter coefficients of multi-resolution analysis).

Promotion the two-scale equation above, define the following recurrence relation [9]:

$$
\begin{aligned}
& \omega_{2 n}(t)=\sqrt{2} \sum_{k \in z} h_{0 k} \omega_{n}(2 t-k) \\
& \omega_{2 n+1}(t)=\sqrt{2} \sum_{k \in z} h_{1 k} \omega_{n}(2 t-k)
\end{aligned}
$$

When $n=0, \omega_{0}(t)=\phi(t)$; when $n=1, \omega_{1}(t)=\psi(t)$. The set of functions $\left\{\omega_{n}(t)\right\} n \in z$ defined above is the Wavelet packet identified by the $\omega_{0}(t)=\phi(t)$. Thus, wavelet packet is a set that has some relation functions including the scaling function $\omega_{0}(t)$ and mother wavelet function $\omega_{1}(t)$. As shown in Fig. 1, it is the diagram of wavelet packet decomposition. $S$ represents original signal, $a_{1}$ represents the 1 st low frequency coefficient $X_{10}$,which decomposed by wavelet packet, $d_{1}$ represents the 1 st low frequency coefficient $X_{11}$, others is so on.

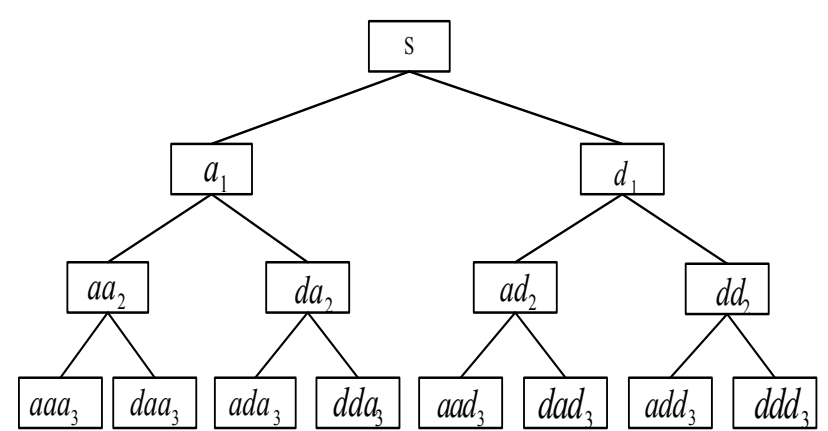

Fig. 1. Signal diagram of wavelet packet decomposition

\section{Wavelet packet energy vector algorithm}

(1) The wavelet packet is adopted to decompose the original signals.

(2) The energy feature of each band

$$
E_{3 j}=\int\left|S_{3 j}(t)\right|^{2} d t=\sum_{1}^{n}\left|x_{j k}\right|^{2}
$$

Where, $\quad x_{j k}(j=0,1, \mathrm{~K}, 7 ; k=1,2 \mathrm{~K}, n) \quad$ represents amplitude of the reconstructed signal.

(3) The wavelet packet energy eigenvector

The definition of all the energy of signal:

$$
E=\sum_{j=0}^{7} E_{3 j}
$$

A band of relative wavelet packet energy:

$p_{3 j}=\frac{E_{3 j}}{E}$

The definition of relative wavelet packet energy feature vector [10]:

$$
K_{i}=\left(p_{30}, p_{31}, \ldots, p_{37}\right)
$$

\section{Elman neural networks}

Recurrent neural networks have feedback links and incorporate temporal aspects better than feedforward neural networks. They also have state variables for the delays, so less information is required when modeling a system. The Elman network is a recurrent neural network of simple architecture which can be trained with the backpropagation algorithm. The Elman network consists of several layers, with the nodes using the input function, the weight function and the transfer functions (activation functions). The first layer is the input layer and the inputs are weighted with the weight function. Besides the output layer, all the other layers have recurrent links [11].

The state units of the Elman network can memorize all the feed inputs such that the outputs of the network depend upon the current input as well as the previous inputs. The state layer of the Elman network makes it different from the multilayer perceptron neural network [12]. 
The network can be expressed as follows[13]:

$$
\begin{aligned}
& x(k)=f\left(w^{1} x_{c}(k)+w^{2}(u(k-1))\right) \\
& x_{c}(k)=x(k-1) \\
& y(k)=g\left(w^{3} x(k)\right)
\end{aligned}
$$

Where, $w^{1}, w^{2}, w^{3}$, are respectively the weight matrices of context units to hidden units, input units to hidden units and hidden units to output units. $f(\cdot)$ And $g(\cdot)$ are nonlinear activation function vector of output units and hidden units respectively.

Fig. 2 shows the Elman neural network structure for fault diagnosis of auxiliary inverter.

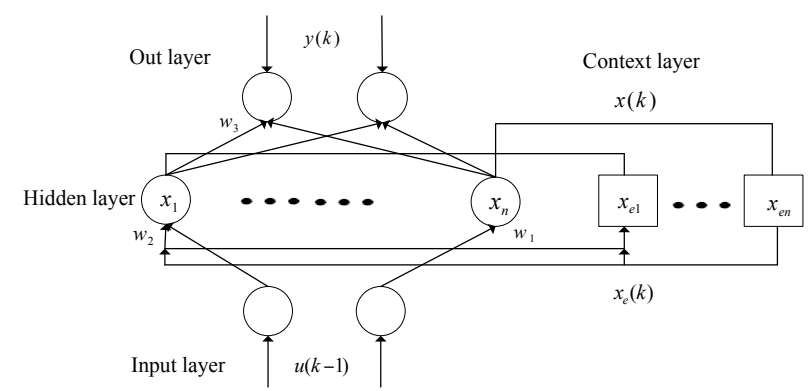

Fig. 2. Framework of Elman neural network

\section{Experimental results}

\subsection{The process of wavelet packet}

Fig. 3(a) - Fig. 5 (a) are the time domain of the signal. For wavelet packet decomposition of the original data, the decomposition structure at 3 is realized and shown in Fig. 3 (b) - Fig. 5(b). After wavelet packet decomposition, the wavelet packet energy eigenvector is constructed. The dataset is divided into two groups, namely: the training and testing group. The training dataset is used for determining the Elman neural network parameters. The testing dataset is used to validate the performance of the trained Elman neural network model. The training group is shown in Tab. 1, the testing group is shown in Tab. 2.

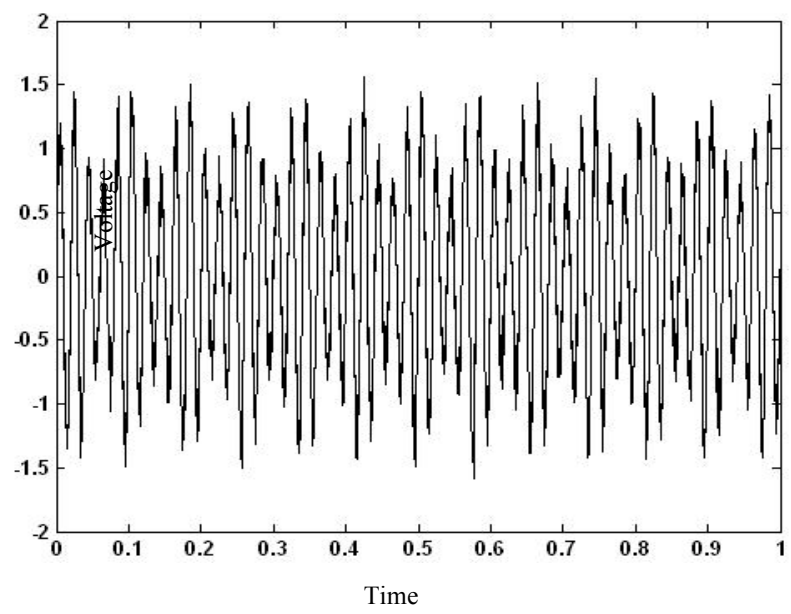

Fig. 3(a). The time domain of voltage fluctuation signal
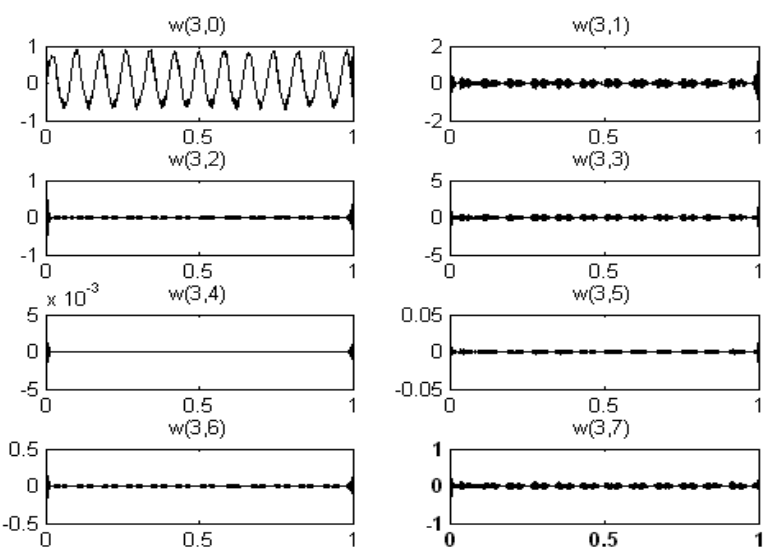

Fig. 3(b). The reconstructed waveform of voltage fluctuation signal

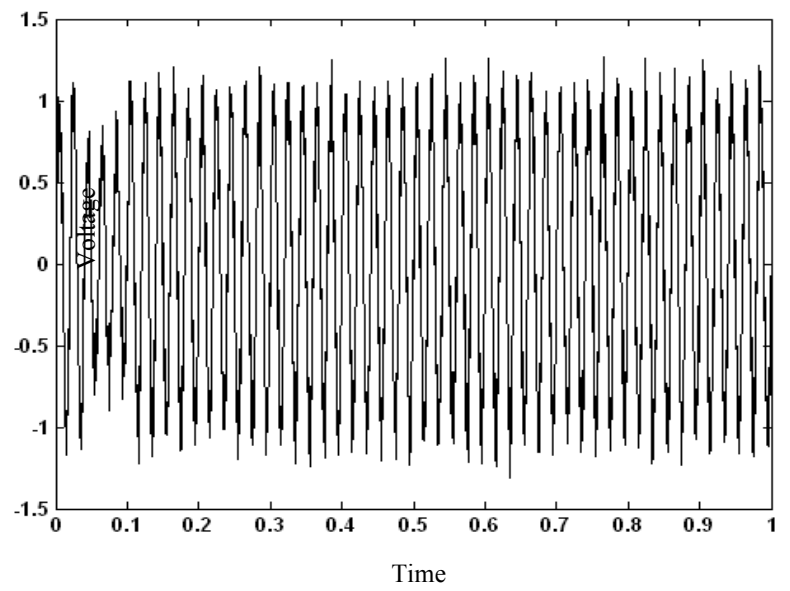

Fig. 4(a). The time domain of power interruption signal
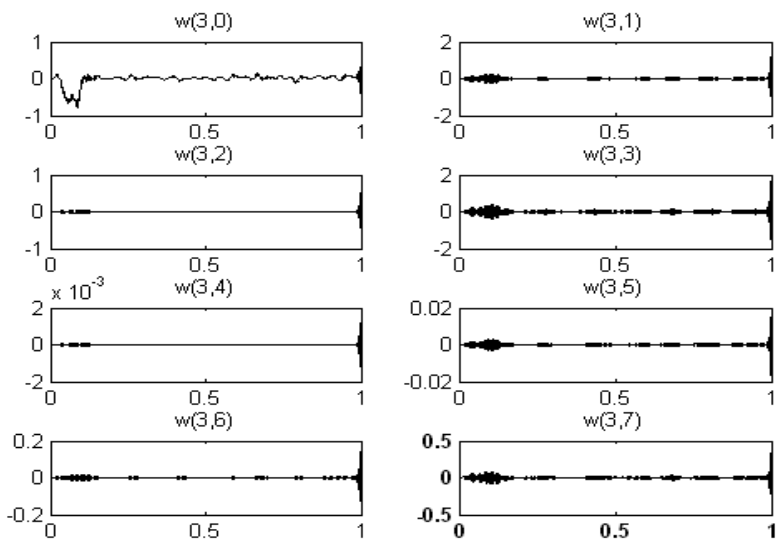

Fig. 4(b). The reconstructed waveform of power interruption signal

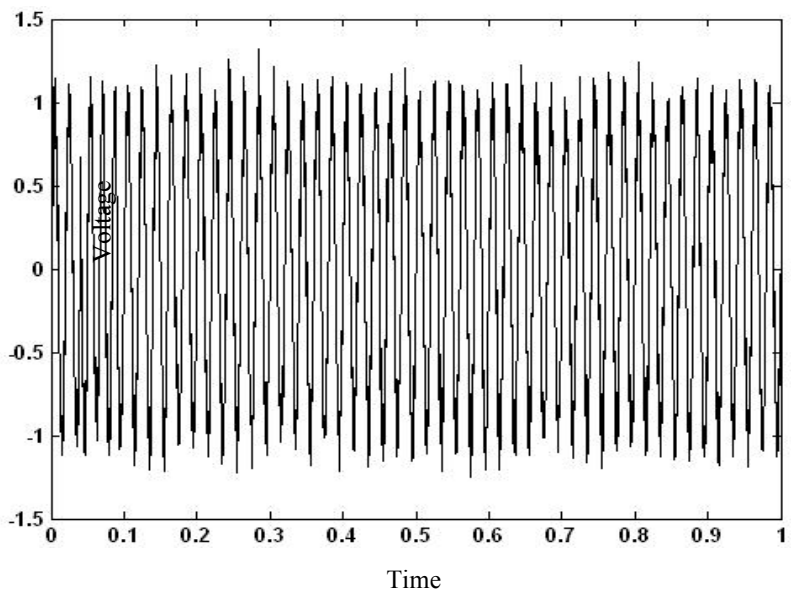

Fig. 5(a). The time domain of frequency variation signal 

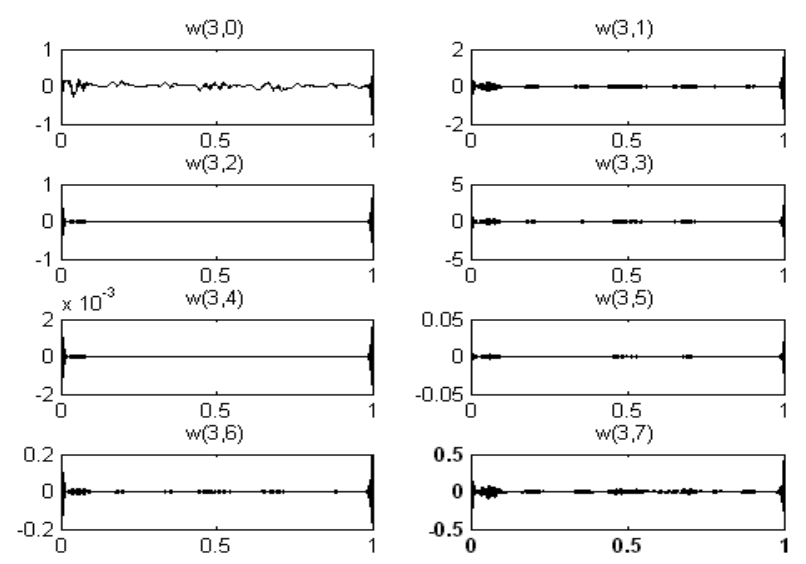

Fig. 5(b). The reconstructed waveform of frequency variation signal

\subsection{BP neural network and Elman neural network for fault diagnosis and classification}

The MATLAB software is used to validate the performance of the proposed approach in this paper. The BP neural network and Elman neural network consists of 8 neurons in the input layer, 3 neurons in the output layer. 8 inputs corresponding to the 8 different ranges of the frequency spectrum of a fault signal, 3 outputs corresponding to 3 respective signals, such as voltage fluctuation signal, impulsive transient signal and Frequency variation signal. After many times of experiments, the hidden layer adopts 7 neurons with faster speed and better learning effect, so the structure of Elman neural network is 8-7-3. The BP neural network is 8-10-3. After trained the network, the testing group is used to examine the trained BP neural network and Elman neural network.
The test results of BP neural network are shown in Tab. 3 and Elman neural network are shown in Tab. 4. By contrasted the outputs of the examination sample with the ideal outputs of the examination sample, the outputs of BP, Elman neural network are all closed to the corresponding ideal outputs of the examination sample, the outputs of Elman neural network are better than BP neural network.

\section{Conclusions}

In this paper, an new method for fault diagnosis and classification in urban rail vehicle auxiliary inverter based on wavelet packet and Elman neural network is presented.

(1) The wavelet packet can subdivide the signal accurately and extract the eigenvector of signal, it can distinguish every fault type.

(2) With memory and recurrent feedback connections, an Elman neural network can learn temporal patterns effectively, and has better robustness.

The experimental results show that the wavelet packet and Elman neural network analysis technology can identify the failure characteristics of the urban rail vehicle auxiliary inverter. So it has fair prospects of application for the inverter fault diagnosis.

\section{Acknowledgements}

This paper was supported by the National Key Technology R\&D Program in the 11th Five year Plan of china (2011BAG01B05), the State Key Laboratory of Rail Traffic Control and Safety (No. RCS2010ZZ002), Beijing Jiaotong University and Doctor Foundation of Shandong Province (BS2011DX008).

Tab. 1. Sample data of urban rail vehicle auxiliary inverter operation

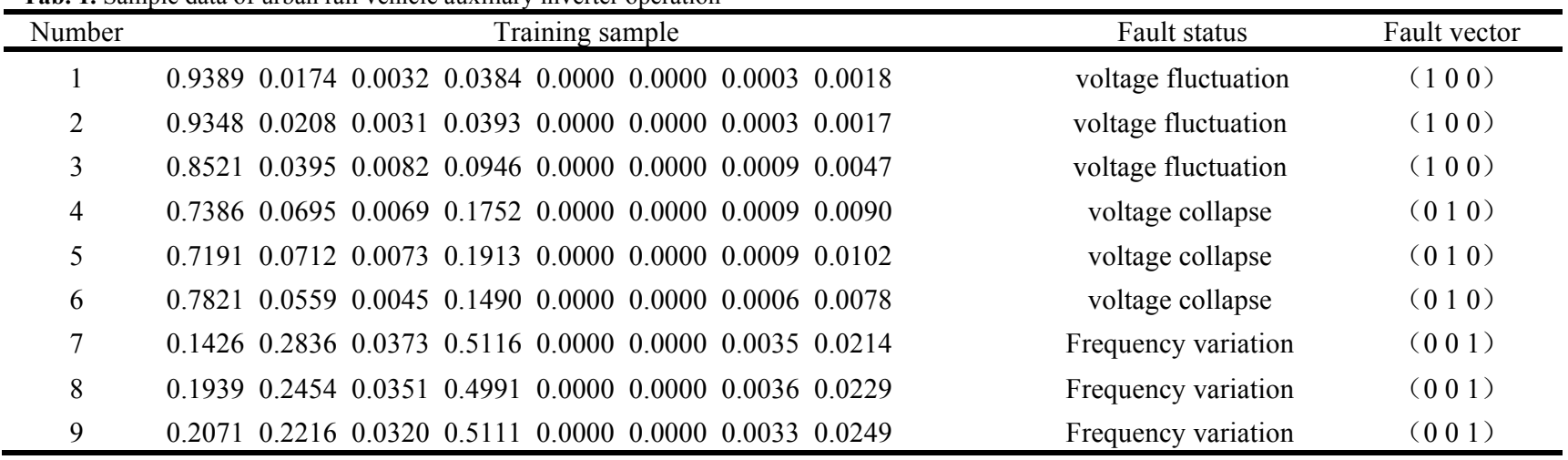

Tab. 2. Testing data

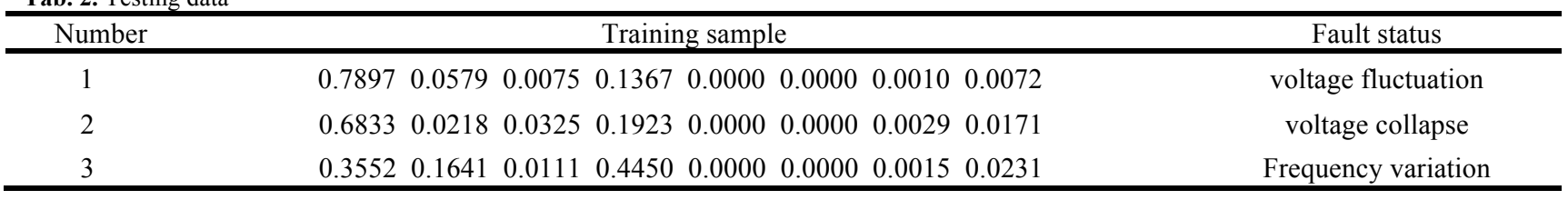

Tab. 3. Testing results of BP neural network

\begin{tabular}{|c|c|c|c|c|c|}
\hline \multirow{3}{*}{$\begin{array}{c}\text { Fault status } \\
\text { voltage fluctuation } \\
\text { impulsive transient }\end{array}$} & \multirow{2}{*}{$\frac{\text { Fault vector }}{\left(\begin{array}{lll}1 & 0 & 0\end{array}\right)}$} & \multicolumn{3}{|c|}{ Actual outputs } & \multirow{2}{*}{$\frac{\text { Testing results }}{\text { voltage fluctuation }}$} \\
\hline & & $(0.9213$ & 0.0179 & $0.0659)$ & \\
\hline & $\left(\begin{array}{lll}0 & 1 & 0\end{array}\right)$ & $(0.0301$ & 0.8912 & $0.0358)$ & voltage collapse \\
\hline Frequency variation & $\left(\begin{array}{lll}0 & 0 & 1\end{array}\right)$ & $(0.0287$ & 0.0231 & $0.8565)$ & Frequency variation \\
\hline
\end{tabular}


Tab. 4. Testing results of Elman neural network

\begin{tabular}{|c|c|c|c|c|c|}
\hline Fault status & Fault vector & \multicolumn{3}{|c|}{ Actual outputs } & Testing results \\
\hline voltage fluctuation & $\left(\begin{array}{lll}1 & 0 & 0\end{array}\right)$ & $(0.9258$ & 0.0124 & $0.0127)$ & voltage fluctuation \\
\hline impulsive transient & $\left(\begin{array}{lll}0 & 1 & 0\end{array}\right)$ & $(0.0301$ & 0.9137 & $0.0242)$ & impulsive transient \\
\hline Frequency variation & $\left(\begin{array}{lll}0 & 0 & 1\end{array}\right)$ & $(0.0220$ & 0.0143 & $0.9516)$ & Frequency variation \\
\hline
\end{tabular}

\section{References}

1. Zhang Lanhong, $\mathrm{Hu}$ Yuwen. Fault Diagnosis and Tolerant Techniques of Inverter in Three-Phase Variable Frequency Drive System, Transactions of china electrotechnical society. 2004,12(19):1-9.

2. Yang Qiaoling, Zhang Haiping. Research on inverter fault diagnosis of brushless DC motor. Industrial instrumentation \& automation. 2012, 1:6-9.

3. Dai Peng, Cai Yun. Fault diagnosis for inverters based on wavelet packet transform and neural network. Power supply technologies and applications, 2011,8(14):17-22.

4. Pratesh Jayaswal, A. K.Wadhwani. Machine Fault Signature Analysis. International Journal of Rotating Machinery. 2008:1-10.

5. Shouxin Ren, Ling Gao. Resolve of overlapping voltammetric signals in using a wavelet packet transform based Elman recurrent neural network. Journal of Electroanalytical Chemistry. 2006, (586):23-30.

6. Muhammad Ardalani-Farsa, SaeedZolfaghari. Chaotic time series prediction with residual analysis method using hybrid ElmanNARX neural networks, Neurocomputing. 2010,(73):2540-2553.
7. Jianda $\mathrm{Wu}$, Jienchen Chen. Continuous wavelet transform technique for fault signal diagnosis of internal combustion engines. NDT\&E International, 2006, (39):304-311.

8. Wenxing Bao, Pu Wang. Romote sensing image fusion based on wavelet packet analysis, Communication Software and Networks. 2011:359-362.

9. Jiang Ling. Rearch of Remote Sensing Image Fusion Based on CurVelet Transform [D]. Harbin Engineering University.2008.

10. Yao Dechen, Yang Jianwei. Fault Diagnosis of Railway Bearing Based on Muti-method Fusion Techniques. Machine Design and Research, 2010,26(3):70-73.

11. W. Zhang, C. Bai, G. Liu, Neural network modeling of ecosystems: a case study on cabbage growth system, Ecol. Model. 2007,201:317-325.

12. S.I. Ao, V. Palade. Ensemble of Elman neural networks and support vector machines for reverse engineering of gene regulatory networks. Applied Soft Computing. 2011,(11):1718-1726.

13. Cai Guoqiang, Yang Jianwei. Fault Diagnosis of Railway Rolling Bearing Based on Wavelet Packet and Elman Neural Network, IASTED 2009:268-274. 